\title{
Feofíceas novas e raras para o litoral brasileiro*
}

\author{
Gilberto M. Amado Filho \\ Laboratório de Algas, Jardim Botânico do Rio de Janeiro, \\ Rua Pacheco Leão 915, 22460-030, Rio de Janeiro, RJ. Brasil. \\ Yocie Yoneshigue-Valentin \\ Instituto de Biologia, Universidade Federal do Rio de Janeiro, \\ Ilha do Fundão, 21944-900, Rio de Janeiro, RJ, Brasil.
}

\begin{abstract}
Resumo
Durante estudo sobre a flora marinha bentônica do litoral de Saquarema a Itacoatiara, Estado do Rio de Janeiro, foram coletadas feoficeas de pequeno porte epifitas em Sargassum filipendula C. Agardh, dentre as quais destacam-se: Myrionema strangulans Greville (Myrionemataceae) como primeira citação da ocorrência do gênero no litoral brasileiro. Hecatonema floridana (Taylor) Taylor (Myrionemataceae) como primeira citação do gênero no litoral sudeste e Elachista minutissima Taylor (Elachistaceae) como primeira citação do gênero no litoral do Estado do Rio de Janeiro.
\end{abstract}

\begin{abstract}
During the study of the benthic marine algae from Saquarema to Itacoatiara (Rio de Janeiro

State) little epiphytic species of Phaeophyta were collected upon thalli of Sargassum filipendula C. Agardh. Among these: Myrionema strangulans Greviille (Myrionemataceae) a new record for the Brazilian coast, Hecatonema floridana (Taylor) Taylor (Myrionemataceae) a new record for the southeast region, and Elachista minutissima Taylor (Elachistaceae) a new record for the Rio de Janeiro State.
\end{abstract}

\section{INTRODUÇÃO}

Este trabalho faz parte de um estudo global sobre a flora marinha bentônica do litoral de Saquarema a Itacoatiara, Estado do Rio de Janeiro (AMADO FILHO, 1991). Este trecho do litoral fluminense apresenta características hidrológicas peculiares por estar próximo (cerca de $50 \mathrm{~km}$ ) ao núcleo do fenômeno sazonal da ressurgência costeira (baixas temperaturas e elevados teores de nutrientes), que ocorre em Arraial do Cabo. Devido à ocorrência deste fe-

\footnotetext{
* Este trabalho faz parte da dissertação de mestrado (Botânica, Museu Nacional, UFRJ) de Gilberto M. Amado Filho

nômeno, essa região tem grande importância biogeográfica (OLIVEIRA FILHO 1977; YONESHIGUE 1985) e características bastante peculiares quanto à flora marinha bentônica (YONESHIGUE 1984; YONESHIGUE E FIGUEIREDO 1983; 1987; YONESHIGHE e OLIVEIRA FILHO 1984; YONESHIGUE $e t$ al. 1986; YONESHIGUE e VILLAÇA 1986).

Durante o inventário da flora marinha bentônica de Saquarema a Itacoatiara, foram coletadas feoficeas de pequeno porte epífitas em Sargassum filipendula C. Agardh, dentre as quais, destacam-se: Myrionema strangulans 
Greville (Myrionemataceae) como primeira citação da ocorrência do gênero no litoral brasileiro, Hecatonema floridana (Taylor) Taylor (Myrionemataceae) como primeira citação do gênero no litoral sudeste e Elachista minutissima Taylor (Elachistaceae) como primeira citação do gênero no litoral do Estado do Rio de Janeiro. Neste trabalho são apresentadas descrições e ilustrações detalhadas das espécies estudadas.

\section{MATERIAL E MÉTODOS}

As algas foram coletadas no costão direito (Ponta da Barra) da Praia de Itaúna e na Laje de Itaúna (Município de Saquarema, fig. 1), no infralitoral, em locais parcialmente protegidos da ação das ondas, onde as populações de Sargassum filipendula C. Agardh apresentavam individuos bem desenvolvidos. Para Myrionema strangulans, a descrição foi complementada com material coletado em Arraial do Cabo (Furnas das Mulheres, fig. 1). 0 material coletado foi triado em água do mar e fixado em formol neutro a $4 \%$.
Para determinação dos táxons, foram aplicados os métodos usuais em Ficologia. Um mínimo de 10 medidas foi efetuado para cada caráter, em pelo menos 5 exemplares de cada táxon. Os desenho foram feitos com auxílio de câmara clara acoplada a microscópio estereoscópico e microscópio óptico.

Para cada táxon é fornecido a obra "princeps"; basiônimo (quando o caso); referências utilizadas no auxílio da identificação; material examinado e número de registro no Herbário RB; descrição detalhada; e comentários sobre características distintivas, limites taxonômicos e comparação com outras descrições.

\section{RESULTADOS E COMENTARIOS}

\section{Chordariales}

Myrionemataceae

Myrionema strangulans Greville

Crypt. Fl., pr. 300, 1827.

Hamel 1931-39, p. 88, fig. 24; Fletcher, 1987, p. 112 , fig. 14.

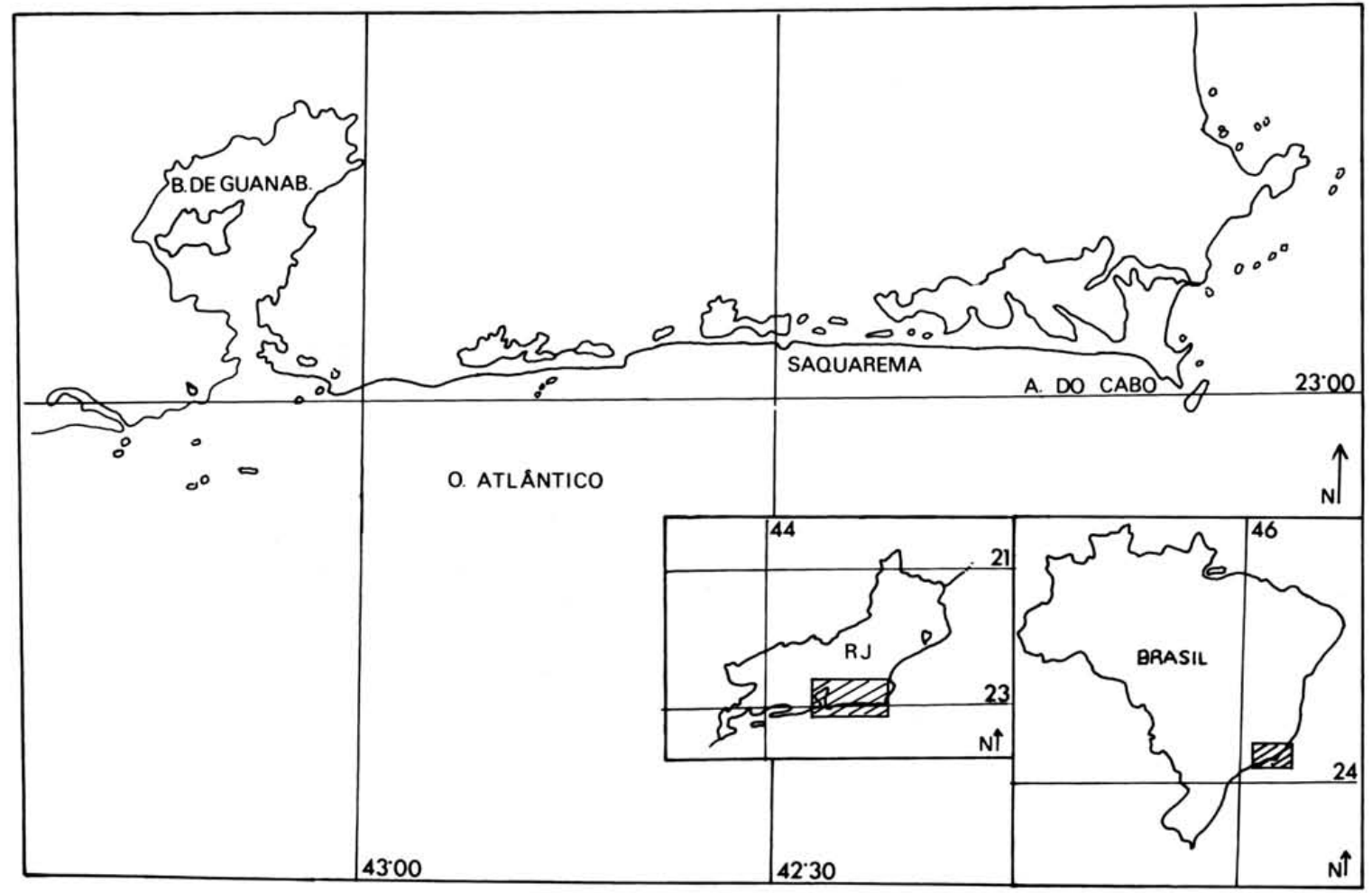

Fig. 1. Mapa com a localização do Município de Saquarema e também de Arraial do Cabo, onde foram encontradas as plantas. 
MATERIAL EXAMINADO: Pta. da Barra 03/09/86 (RB 200912), Furnas das Mulheres $05 / 09 / 90$.

Figuras 2 a 6.

Plantas epífitas em forma de pequenas manchas arredondadas, cor marron, 0,5 a $1,0 \mathrm{~mm}$ de diâmetro, coberta por longos pêlos, composta por filamentos decumbentes e eretos, esses últimos decrescendo de tamanho do centro para a periferia.

Em vista superficial, o talo rastejante monostromático é composto por fileiras radiais de células frouxamente unidas, que medem de 4 a $10 \mu \mathrm{m}$ de comprimento, as células apicais frequentemente dividindo-se por dicotomia (fig. 2).

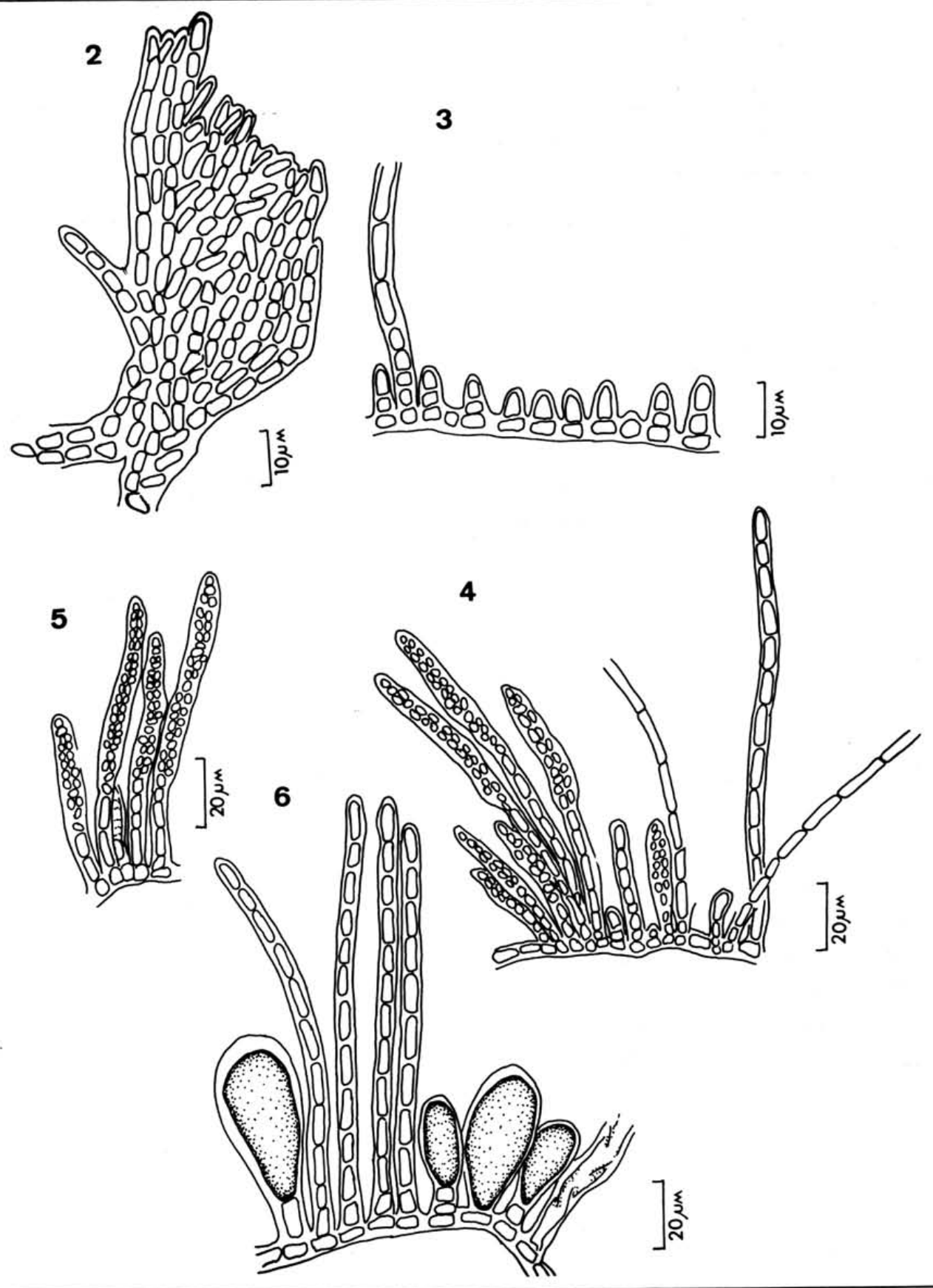

Fig. 2. Vista superficial do talo rastejante monostromático. Fig. 3. Vista lateral da porção marginal do talo. Fig. 4. Vista lateral do talo, mostrando hipotalo, filamentos eretos, pêlos e órgãos pluriloculares. Fig. 5. Detalhe dos órgãos pluriloculares. Fig. 6. Detalhe dos órglios uniloculares. 
Em corte transversal, a região basal ou hipotalo é uniestratificada, sem rizóides, formada por células mediando de 4 a $11 \mu \mathrm{m}$ de comprimento e 3 a $5 \mu \mathrm{m}$ de largura (figs. 3, 4, 5 e 6). Cada célula hipotaliana emite geralmente um filamento ereto, as vezes dois, simples (figs. 4 e 6). Os filamentos adultos são formados por 10 a 13 células, atingindo todos a mesma altura ( $130 \mu \mathrm{m}$ de comprimento e 5 a $6 \mu \mathrm{m}$ de largura) (figs. 4 e 6). As células contem 2 a 3 plastídios em forma de placa. Os pêlos são incolores, de crescimento basal, originados das células do hipotalo (fig. 4).

Esporângios pluriloculares clavados, uni a bisseriados, 18 a $70 \mu \mathrm{m}$ de comprimento e 5 a $8 \mu \mathrm{m}$ de largura, inseridos diretamente no estrato basal ou terminais nos filamentos eretos (figs. 4 e 5 ).

Esporângios uniloculares piriformes, às vezes alongados, 26 a $45 \mu \mathrm{m}$ de comprimento e 12 a $25 \mu \mathrm{m}$ de largura, nascendo diretamente da região hipotaliana, sésseis ou com pedicelo unicelular (fig. 6).

Plantas estéreis e com orgão pluriloculares foram coletadas sobre filóides de S. filipendula na Pta. da Barra (Saquarema), e plantas com órgãos uniloculares junto a Stragularia clavata (Harvey in Hooker) Hamel na região de Arraial do Cabo.

COMENTÁRIOS: Pelas características talo epifitico que forma pequena mancha circular sobre o hospedeiro, com a base discóide monostromátia, da qual se originam filamentos eretos não ramificados de sua parte central, os exemplares ora estudados enquadram-se no gênero Myrionema Greville (FLETCHER, 1987).

Pelos filamentos eretos com 5 a $6 \mu \mathrm{m}$ de largura, esporângios pluriloculares, clavados, frequentemente bisseriados e medindo de 18 a $70 \mu \mathrm{m}$ de comprimento, as referidas plantas concordam com $M$. strangulans Greville (HAMEL 1931-39, FLETCHER 1987). Embora haja concordância no seu contexto geral morfológico, o material que estudamos apresenta orgãos pluriloculares pouco maiores que os citados por FLETCHER (1987) para as Ilhas Britânicas, onde alcançam até $50 \mu \mathrm{m}$ de comprimento; também a altura dos filamentos vegetativos do presente material é superior (130 $\mu \mathrm{m})$ à das plantas citadas por HAMEL (193139) para a costa francesa $(100 \mu \mathrm{m})$.

Os presentes orgãos uniloculares são semelhantes na forma e no tamanho ao das plantas citadas anteriormente para a Europa, entretanto, nascem diretamente das células hipotalianas e não da base dos filamentos vegetativos, como nos exemplares em FLETCHER (1987).

Hecatonema floridana (Taylor) Taylor Mar. Algae Trop. Subtrop. Amer., p. 241, 1960. Basiônimo: Phycocoelis floridana Taylor, Carnegie Inst. Wash. Publs., 379:109, 1928. Taylor 1960 , p. 241; Joly et al. 1969, p. 64, figs. 1621 .

MATERIAL EXAMINADO: Pta. da Barra 03/09/86 (RB200911), 18/03/87.

Figuras 7 a 11 .

Plantas epifitas, formando pequenos tufos de cor marron-esverdeadas, atingindo até $1 \mathrm{~mm}$ de altura.

O talo é formado por filamentos decumbentes, ramificados, densamente distribuidos sobre o talo do hospedeiro. Os filamentos são formados, em quase todo sua extensão, por duas camadas de células (figs. 7, 8, 10 e 11). Esses filamentos originam filamentos eretos, unisseriados, simples ou raramente ramificados com 8 a $12 \mu \mathrm{m}$ de diâmetro e células com o comprimento de 1 a 3 vezes o próprio diâmetro (figs. 7, 8, 9 e 10), plastídios discóides. Presença de pêlos hialinos, longos, com crescimento basal, cerca de $8 \mu \mathrm{m}$ de diâmetro, originados dos filamentos decumbentes (fig. 9).

Orgãos pluriloculares fusiformes (figs. $7 \mathrm{e}$ 8), ovóides quando jovens (fig. 9), com (44) 78 (96) $\mu \mathrm{m}$ de comprimento e (18)24(30) $\mu \mathrm{m}$ de diâmetro, sésseis ou pedunculados nos eixos prostrados, ou terminais nos filamentos eretos.

COMENTÁRIOS: Por apresentar filamentos decumbentes, com um, ou mais geralmente, duas camadas de células, densamente distribuidos, filamentos eretos unisseriados, simples ou pouco ramificados, plastídios discóides. pêlos com crescimento basal, originados dos filamentos decumbentes, esporângios pluriloculares, originados tanto da porção basal quanto da terminal nos filamentos eretos, as atuais plantas estão de acordo com a circunscrição do gênero Hectonema Sauvage in TAYLOR (1960). Medidas dos filamentos e pêlos, bem como 
forma e dimensões dos orgãos pluriloculares estão de acordo com as de Hecatonema floridana (Taylor) Taylor (TAYLOR 1960; JOLY et al 1969). A ocorrência desta espécie no litoral brasileiro encontrava-se limitada ao Arquipélago dos Abrolhos, Bahia (JOLY et al 1969).
Elachista minutissima Taylor

Pap. Tortugas Lab., 25:113, 1928.

Taylor 1928, p. 113, pr. 14, fig. 17; 1960; p. 245 , pr. 29 , fig. 11.

MATERIAL EXAMINADO: Ponta da Barra 18/02/87 (RB200914), Laje de Itaúna 06/06/87 (RB200913).

\section{Elachistaceae}

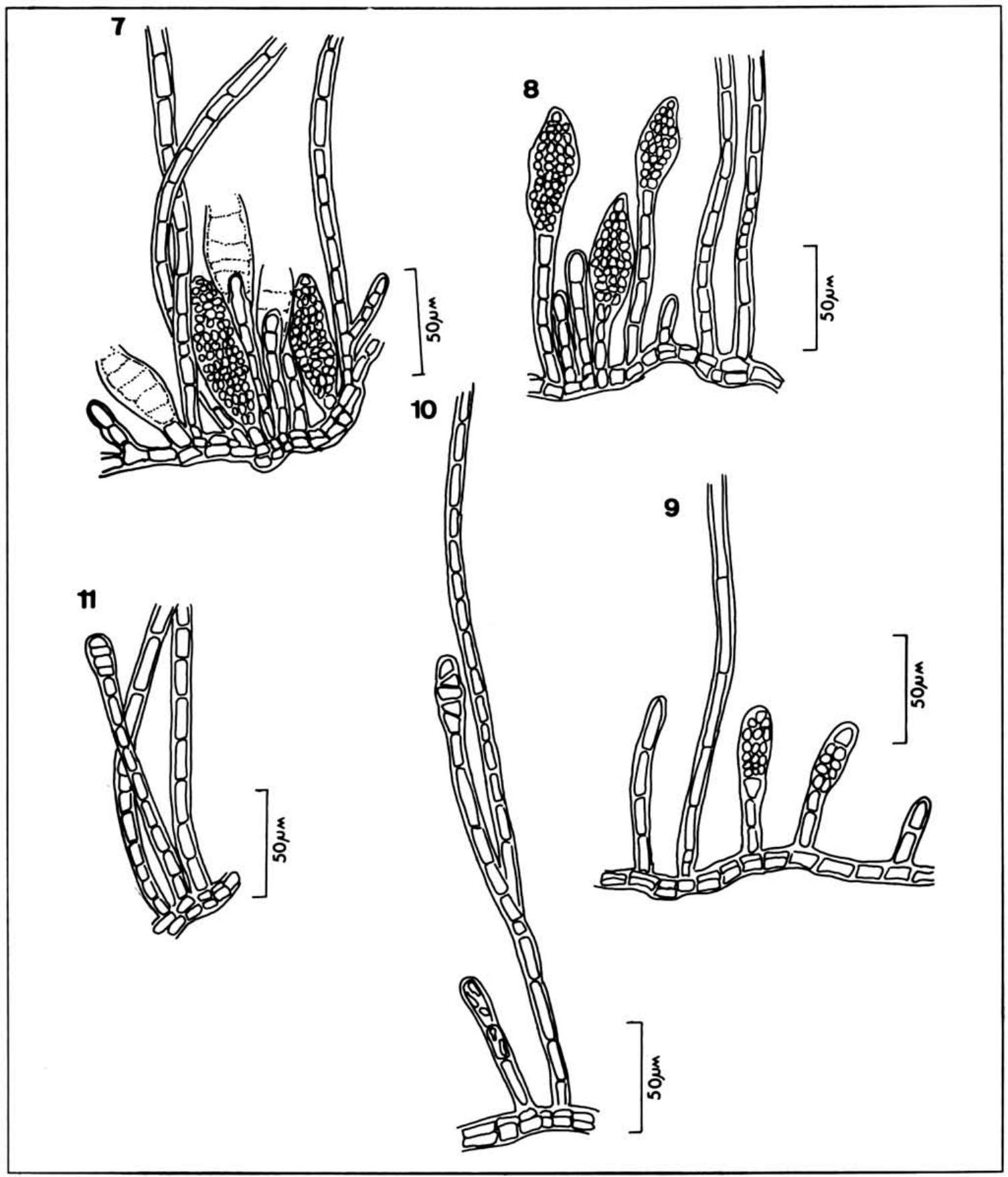

Figuras 7 e 8. Vista lateral do talo, mostrando filamentos decumbentes, filamentos eretos e órgãos pluriloculares. Fig. 9. Vista lateral do talo, mostrando filamento decumbente, fílamento ereto, pêlo, e órgãos pluriloculares em desenvolvimento. Fig. 10. Parte do talo, mostrando filamento decumbente, filamentos eretos e detalhe dos plastos. Fig. 11. Detalhe do filamento decumbente com duas camadas de células. 


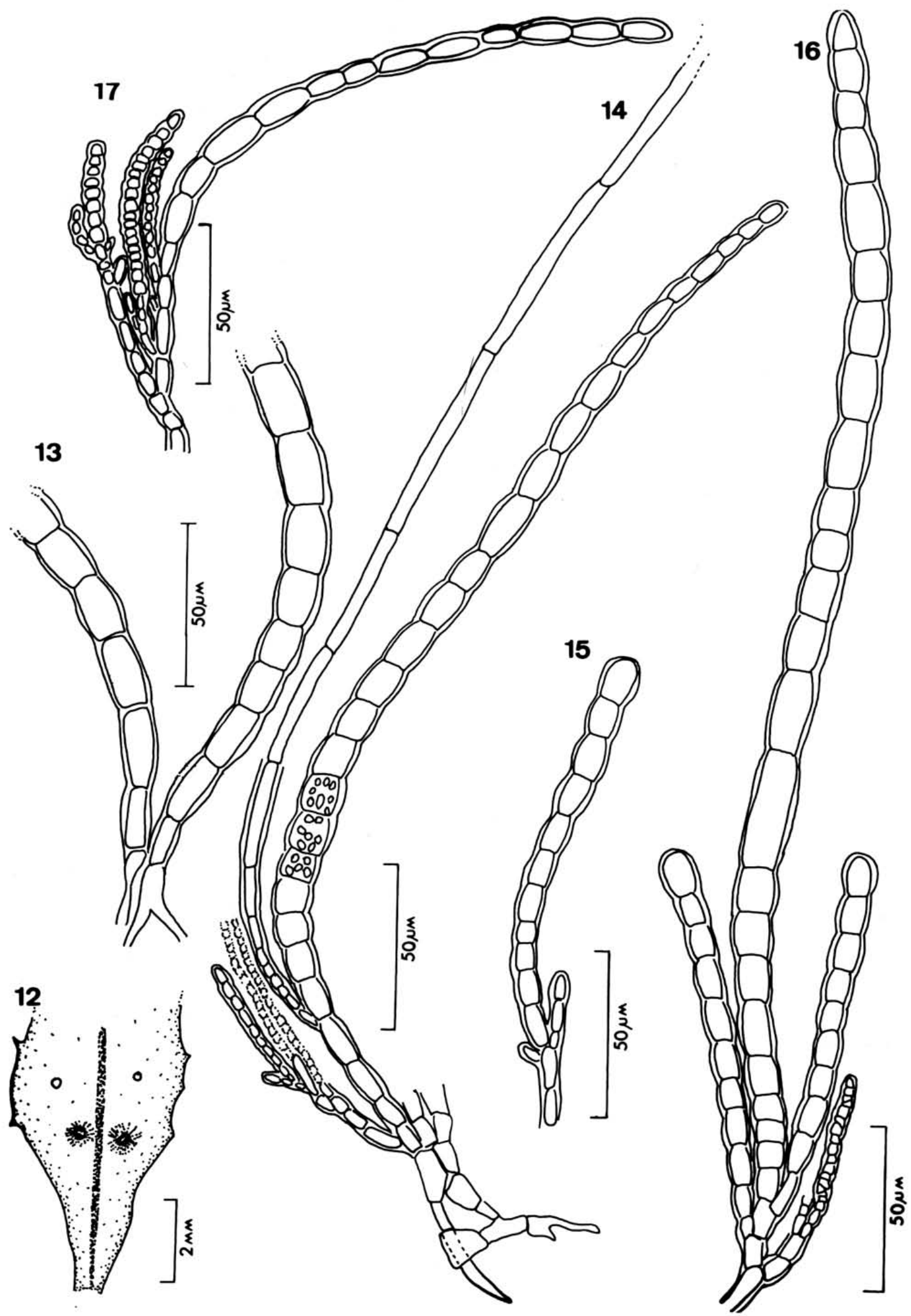

Figura 12. Hábito da planta, epífita em Sargassum filipendula. Fig. 13. Detalhe da porção basal do filamento. Fig. 14. Parte do talo, mostrando porção basal, filamento longo, pêlo, gamentângios e detalhe dos plastos. Fig. 15. Detalhe da paráfise. Fig. 16. Parte do talo, mostrando filamento longo, paráfises e gametângios. Fig. 17. Detalhe dos gametângios. 
Figuras 12 a 17

Talo em forma de pulvínulo, de cor marrom, atingindo até $1,5 \mathrm{~mm}$ de altura.

O talo é formado por uma porção basal, filamentos livres (longos e curtos) e gametângios. Os filamentos longos determinam o aspecto hemisférico do talo (fig. 12), enquanto que os curtos e os gametângios causam-lhe uma linha sombreada.

A porção basal é caracterizada por filamentos incolores muito emaranhados (figs. 13 e 14), penetrando nos criptóstomas do hospedeiro. Deste estrato basal, partem dois tipos de filamentos, simples, ambos com células contendo numerosos plastídios discóides. Os filamentos curtos, denominados paráfises, dispostos concentricamente, atingem quase todos a mesma altura, são de aspecto claviforme e formados por 10 a 15 segmentos moniliformes com 7 a $10 \mu \mathrm{m}$ de diâmetro (figs. 14,15 e 16). Os filamentos longos (assimiladores) são formados por segmentos cilíndricos, atenuados na base, moniliformes no sentido do ápice e com 5 a $14 \mu \mathrm{m}$ de diâmetro (figs. 14,16 e 17). 0 crescimento é intercalar ao longo de todo o talo, conferindo comprimento variável aos segmentos.

Os filamentos assimiladores emitem na base pêlos de crescimento basal e gametângios (figs. 14 e 17). Esses últimos formam pequenos tufos, são cilíndricos, unisseriados, não ultrapassam a altura das paráfises e medem de 6 a $7 \mu \mathrm{m}$ de diâmetro e (39)64(99) $\mu \mathrm{m}$ de comprimento. Não foram observados órgãos uniloculares.

Os espécimes desta espécie crescem no interior dos criptóstomas de Sargassun filipendula.

COMENTÁRIOS: Talo em tufo, composto por uma porção basal formada por filamentos incolores, entrelaçados, de onde se elevam filamentos livres, longos e curtos, de crescimento intercalar, conferindo aspecto pulvinado à planta são características essenciais da família Elachistaceae. $\mathbf{O}$ estrato basal penetrando no hospedeiro, com filamentos curtos ou paráfises acompanhado os órgãos de reprodução na base dos filamentos assimiladores, são características que distinguem o gênero Elachista Duby dos demais que compõem a família. Os presentes exemplares concordam com os de Elachista minutissima descritos em TAYLOR (1928) para as Ilhas Tortugas, Mar do Caribe, pelas seguintes características: hospedeiro, medidas dos filamentos, diâmetro das paráfises, presença de pêlos verdadeiros e medidas dos gametângios.

Esta espécie foi mencionada por OLIVEIRA FILHO et al (1979) como ocorrendo sobre Sargassum flutuantes, encontrados na corrente do Brasil e, mais tarde, confirmada por um dos autores (E.J. PAULA, com. pessoal) sobre populações fixas de Sargassum em São Sebastião, no Estado de São Paulo.

\section{REFERÊNCIAS BIBLIOGRÁFICAS}

AMADO FILHO, G.M. 1991. Algas marinhas bentônicas do litoral de Saquarema a Itacoatiara (RJ). Dissertação de Mestrado, Universidade Federal do Rio de Janeiro, 324 p., il.

FLETCHER, R.L. 1987. Seaweeds of the British Isles, vol. 3, Fucophyceae (Phaeophyceae) part. 1. British Museum (Natural History), London, 359 p., il.

HAMEL, G. 1931-39. Phaeophycées des France. Paris, 431 p., il.

JOLY, A.B.; UGADIM, Y.; DIAZ, J.J.; YAMAGUISHI-TOMITA, N.; CORDEIROMARINO, M. \& YONESHIGUE-BRAGA, Y. 1969. Additions to the marine flora of Brazil - X. Rickia, 4: 61-74, il.

OLIVEIRA FILHO, C. C. 1977. Algas Marinhas Bentônicas do Brasil. Tese de Livre Docência, Univ. de São Paulo, 407 p., il.

OLIVEIRA, E.C.; UGADIM, Y. \& PAULA, E.J.1979. Comunidades associadas a plantas de Sargassum flutuantes em águas da corrente do Brasil - considerações biogeográficas. Bol. Bot. Univ. S. Paulo, 7:5-9, il.

TAYLOR, W.R. 1928. The marine algae of Florida, with special reference to Dry Tortugas. Pap. Tortugas Lab., Carnegie Inst. Wash., 25: 1-219, il.

1960. Marine algae of the eastern tropical and subtropical coast of the America. Univ. Michigan Press, Ann Arbor, 870 p., il YONESHIGUE, Y. 1984. Flore marine de la region de Cabo Frio (Brésil). 4. Sur une espece nouvelle du genre. Peyssonnelia (Cryptonemiales, Rhodophyta). Vie Milieu, 34(23):133-37, il.

1985. Taxonomie et ecologie des algues marines dans la region de Cabo Frio 
(Rio de Janeiro, Brésil). These de Docteur d'État-Sciences, Faculté de Sciences de Luminy, Univ. D'Aix-Marseille II, France, 466 p. il.

YONESHIGUE, Y.; BOUDORESQUE, C.F.; FIGUEIREDO, M.A.O. 1986. Flore algale marine de la region de Cabo Frio, État de Rio de Janeiro (Brésil), 5-sur Boodlea composita (Boodleaceae-Chlorophyta), Dictyota pardalis (Dictyotaceae-Phaeophyta) et Lophosiphonia cristata (Rhodomelaceae-Rhodophyta), especes nouvelles por la côte brésiliennes. Rickia, 13:1727 , il.

YONESHIGUE, Y. \& FIGUEIREDO, M.A.O. 1983. Flore marine de la region de Cabo Frio (Brésil). 3. Ectocarpaceae (Phaeophyta) nouvelle pour la côte brésilienne. Vie Milieu, 33(3-4): 18190 , il.
1987. Flora marinha da região de Cabo Frio (Estado do Rio de Janeiro, Brasil). 1. Chlorophyceae raras para o litoral brasileiro. Neritica, 2: 119-34, il.

YONESHIGUE, Y. \& OLIVEIRA FILHO, E.C. 1984. algae from Cabo Frio upwelling area. 2. Gelidiocolax pustulata (Gelidiaceae, Rhodophyta), an unusual new putative parasitic species. J. Phycol., 20(3): 440-45, il. YONESHIGUE, Y. \& VILLAÇA, R.C. 1986. Flora marinha da região de Cabo Frio (Estado do Rio de Janeiro, Brasil). 6. Pterosiphonia spinifera, Polysiphonia eastwoodae, $P$. flacidissima, P. sphaeorocarpa, Strebocladia corymbifera (Rhodomelaceae, Rhodophyta), novas ocorrências para a costa brasileira. Rickia, 13: 97-111, il.

Recebido para publicação em 13/05/92. 